\title{
Prozessstrategien zur optimalen Reinigungsfähigkeit am Beispiel des Titus-Zentrifugentrockners TZT 400
}

\author{
Sebastian Stahl*, Johannes Ruland und Hermann Nirschl
}

Häufige Produktwechsel und immer kürzere Produktionszyklen stellen hohe Anforderungen an die Reinigungsfähigkeit von verfahrenstechnischen Maschinen und Apparaten. Vor allem in der Pharma- bzw. der Lebensmittelindustrie ist die Vermeidung von Kreuzkontamination von besonderer Bedeutung. Einige Apparate verbinden zwei Grundoperationen (Unit-Operations) in einem Verfahrensraum, wodurch sich sowohl die Handhabung als auch der Gesamtreinigungsaufwand reduziert. Der Titus-Zentrifugentrockner verbindet die mechanische Entfeuchtung mit einer nachgeschalteten thermischen Trocknung. Der letzte Verfahrensschritt beinhaltet eine „Cleaning in Place“ (CIP)-Prozedur, die von allen vorangegangenen Verfahrensschritten maßgeblich beeinflusst wird. Im Mittelpunkt dieser Arbeit steht die Bestimmung der optimalen Verfahrensparameter, die einerseits die sichere Verarbeitung von schwierigen Produkten ermöglicht und zum anderen die Grundlage für eine effiziente Reinigung liefert. Da eine validierte Reinigungsprozedur in pharmazeutischen Anwendungen, beispielsweise bei toxischen oder hochaktiven Produkten, einen wesentlichen Teil der Gesamtprozesszeit einnimmt, müssen alle Verfahrensschritte direkt aufeinander abgestimmt sein.

Schlagwörter: Hygienic Design, Reinigung, Zentrifugentrockner

Eingegangen: 31. Juli 2007; akzeptiert: 03. August 2007

\section{Einleitung}

Der Titus-Zentrifugentrockner TZT der Firma FIMA Maschinenbau GmbH aus dem HEINKEL Process Technologie Firmenverbund vereinigt die Fähigkeiten einer klassischen, horizontalen, diskontinuierlichen Zentrifuge mit denen eines thermischen Trockners in einem Verfahrensraum. Die Baugrößen des TZT weisen Trommeldurchmesser von $400 \mathrm{~mm}$ bis hin zu $1300 \mathrm{~mm}$ auf. Diese Anlagen eignen sich auch für Produkte mit breiten Partikelgrößenverteilungen. Der TZT besitzt aus Gründen der einfacheren Reinigbarkeit keinerlei Einbauten. Durch ein integriertes PAT (Process Analytical Technology)-System können kritische Prozessparameter während des Prozesses abgefragt werden. Die Integration von neu entwickelten Merkmalen, wie eine Inlineprobennahme, die Restfeuchtebestimmung mittels NIR (Nah-Infrarot)-Spektroskopie, ein integriertes Kamerasystem sowie ein Endoskop, ermöglichen eine Onlineprozessüberwachung. Darüber hinaus gewährleisten zahlreiche konstruktive Feinheiten eine GMP (Good Manufacturing Practice)-konforme und hygienische Produktion.
Das Ziel dieser Arbeit bestand darin, die einzelnen Prozessparameter so zu optimieren, dass eine nachfolgende Reinigung der Anlage effizient, sicher und in möglichst kurzer Zeit vollzogen werden kann. Zur Befüllung gelangt die Suspension im Zentrifugentrockner durch eine mitrotierende Hohlwelle in die Filtertrommel. Der Feststoff wird dabei im Zentrifugalfeld durch ein mehrlagiges Filtergewebe zurückgehalten. Im nächsten Schritt erfolgt die Waschung des Feststoffhaufwerks. Die anschließende mechanische Entfeuchtung erfolgt unter anderem auch durch einen überlagerten Gasdruck.

Im Folgenden sind das klassische Verfahren mit Kuchenabwurf und das neu entwickelte „Gegenimpulsverfahren“, bei dem der Kuchen nach der mechanischen Entfeuchtung als Produktring bestehen bleibt, $\mathrm{zu}$ unterscheiden. Im klassischen Verfahren erfolgt nach der mechanischen Entfeuchtung der Abwurf des Produktkuchens mit anschließender thermischer Trocknung des Haufwerks in der Wirbelschicht. Dabei strömt Heißgas durch Gasimpulse filtratseitig durch das Filtergewebe. Bei einer erwünschten Festbetttrocknung wird der Prozessschritt des Kuchenabwurfes ausge- 


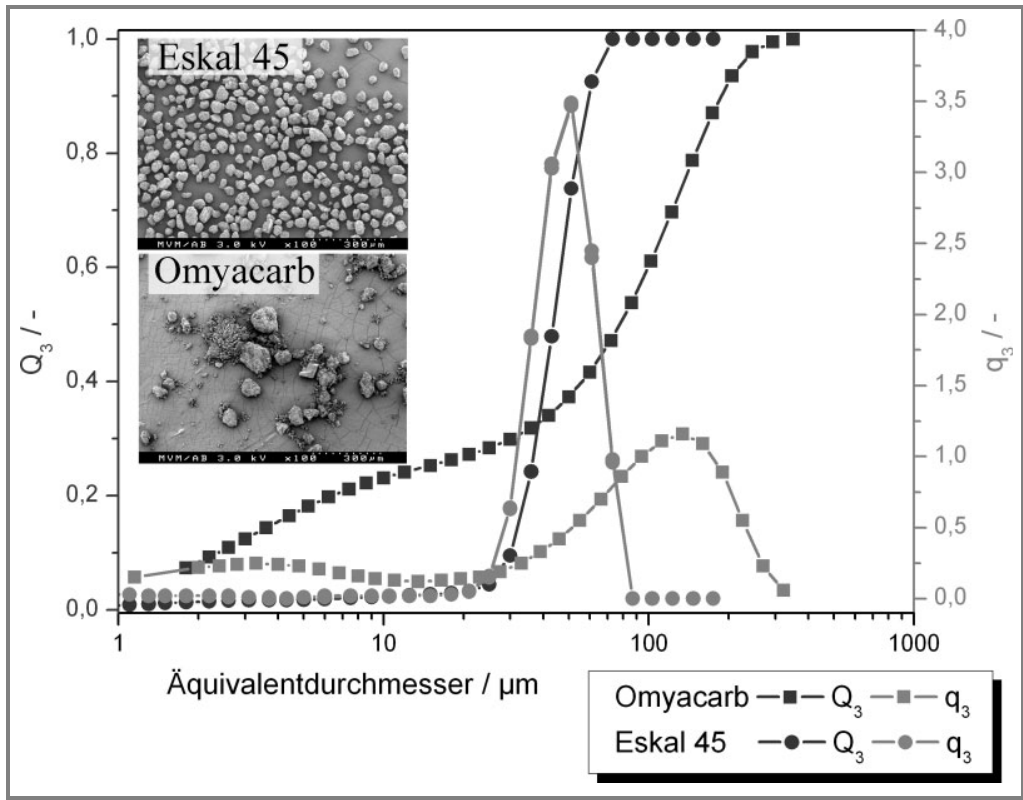

Abbildung 1. Summen- und Dichtenverteilung (Helos) von Omyacarb und Eskal 45.

spart. Die Trocknung des Festbettes gelingt durch Einleitung von heißem Trockengas durch das Füllrohr. Dabei durchströmt das Trocknungsgas das Haufwerk in Filtratrichtung und belädt sich mit der im Haufwerk verbliebenen Feuchte. Dieses Verfahren kommt hauptsächlich bei Stoffen zur Anwendung, die zu Agglomeratbildung neigen.

Um die Prozessschritte zu analysieren werden Pilotversuche des klassischen denen des Gegenimpulsverfahrens gegenübergestellt Die in den Experimenten verwendeten Produkte (Omyacarb, Eskal 45 und Eskal 150) bestehen aus verschiedenen Kalksteinfraktionen mit einer Dichte von $2,7 \mathrm{~g} / \mathrm{cm}^{3}$ (s. Abb. 2). Sie

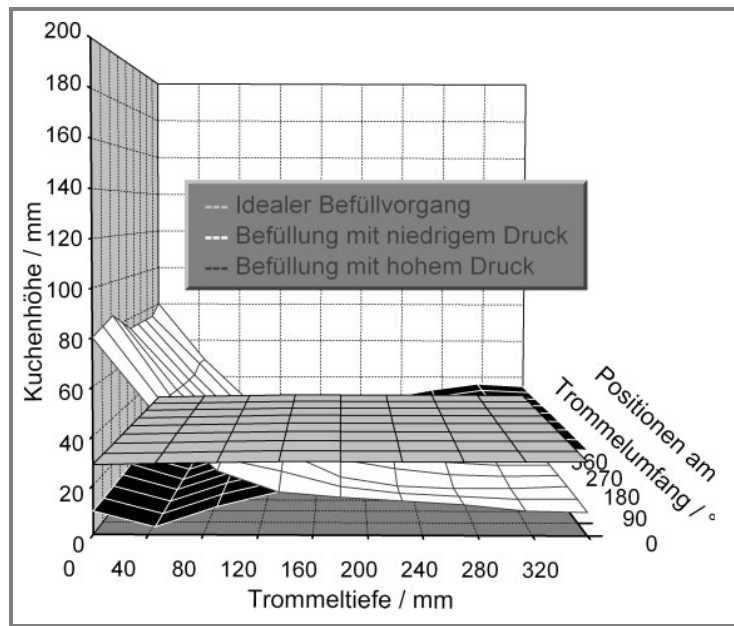

Abbildung 2. 3D-Diagramm der Kuchenstruktur bei unterschiedlichem Fülldruck ermittelt mit einem Ultraschallsensor. Das Kuchenvolumen ist bei den drei dargestellten Kuchen gleich. weisen in ihrer Größenverteilung und ihrem Sedimentationsverhalten Ähnlichkeiten mit häufig verwendeten Produkten aus der Pharmaindustrie bzw. der Feinchemie auf. Zudem sind die Kalksteinfraktionen gesundheitlich unbedenklich.

Wegen seines höheren Feingutanteils und der damit verbundenen Deckschicht stellt Omyacarb ein relativ schwieriges Produkt für die mechanische Entfeuchtung dar. Medianwerte der Massensummenverteilung $\left(x_{50}\right.$-Werte) liegen für das bimodale Omyacarb bei 76,9 $\mu \mathrm{m}$ und für Eskal 45 bzw. Eskal 150 bei $43,71 \mu \mathrm{m}$ bzw. $134,57 \mu \mathrm{m}$.

\section{Charakterisierung der Verfahrensschritte}

Füllen

Beim Befüllvorgang des Verfahrensraumes liegt ein besonderes Augenmerk auf der Drehzahl und dem eingestellten Befüllungsdruck im Rührkessel. Erfahrungen haben gezeigt, dass auch bei schwierigen Produkten sehr gute Verfahrensergebnisse mit einer Fülldrehzahl von $300 \mathrm{U} / \mathrm{min}$ erzielt werden, die einer Zentrifugalbeschleunigung von ca. $20 \mathrm{~g}$ entspricht. Neben der Umdrehungszahl beim Befüllen ist auch der Fülldruck der Suspension für einen guten Kuchenaufbau entscheidend. Der Druck wird über einen Rührkessel eingestellt und die Suspension gelangt danach über ein eingebautes Tauchrohr durch das Füllrohr in die Filtertrommel. Vorteil dieser Methode ist, dass die Auftreffstelle der Suspension in der Trommel durch den aufgegebenen Fülldruck definiert eingestellt werden kann. Erfolgt eine Befüllung aus einem Behälter unter atmosphärischem Druck, so ändert sich die Auftreffstelle der Suspension in der Filtertrommel über der Zeit mit abnehmender Füllhöhe. Zur Erzeugung eines homogenen Kuchens ist deshalb eine drucküberlagerte Befüllung besser geeignet als eine geodätische Befüllung [1].

$\mathrm{Zu}$ hoher wie auch zu niedriger Druck führen bei der Befüllung zu einem inhomogenen Kuchenaufbau (s. Abb. 2). Waschprozesse, mechanische Entfeuchtung und das Abreinigen des Haufwerks gestalten sich dadurch erheblich schwieriger. Als Folge der mechanischen Entfeuchtung sind Haufwerksbereiche zu beobachten, die gar keine oder unzureichende Entfeuchtungsergebnisse aufweisen. Ist der Druck im Rührkessel zu hoch eingestellt, trifft die Suspension vom Füllrohr (s. Abb. 2 links) an die Stauscheibe (s. Abb. 2 rechts). Dadurch kann eine Zerstörung empfindlicher Produkte erfolgen. Man erhält einen inhomogenen Haufwerksaufbau, der eine inhomogene me- 
chanische Entfeuchtung nach sich zieht [1]. Die Ermittlung der Restfeuchte über die Probeentnahme stellt sich ebenfalls schwierig dar, da die Probe nur unzureichend repräsentativ für das gesamte Haufwerk ist. Experimente haben gezeigt, dass bei Suspensionen von 16,6 Gew.-\% bei einem Fülldruck von über 0,45 bar inhomogene Kuchen entstehen.

Erfolgt die Befüllung mit $\mathrm{zu}$ niedrigem Druck, so bildet sich das Haufwerk an der Trommelrückwand nahe dem Füllrohr „bergartig" aus und das Haufwerk entfeuchtet ebenfalls inhomogen. Diese Art der Befüllung ergibt sich bei einer Konzentrationen von 16,6 Gew.-\% Omyacarb, wenn mit einem Fülldruck von unter 0,09 bar befüllt wird.

Beide extreme Befüllarten vermindern die Effizienz der nachfolgenden Prozessschritte. Der Filterkuchen kann mit der falschen Druckeinstellung nur unzureichend gewaschen werden, da dickere Kuchenbereiche nur schwer mit Waschflüssigkeit in Kontakt kommen. Des Weiteren ist eine Verminderung der Wirksamkeit der nachfolgenden Entfeuchtung mit überlagertem Gasdifferenzdruck die Folge, da das Gas immer dem Weg des geringsten Haufwerkswiderstands folgt. Der Abwurf von inhomogenen Haufwerken dauert länger, da zuerst die Bereiche abgeworfen werden, die die geringste Höhe aufweisen.

Zur Ermittlung des Fortschrittes der mechanischen Entfeuchtung spielt der Filtratstrom eine wesentliche Rolle und ist von folgenden Punkten abhängig: dem Produkt, der Suspensionsfeststoffkonzentration, der Partikelgröße und deren Verteilung, der Suspensionsmasse, der Umdrehungszahl der Trommel, des eventuellen beaufschlagten Gasüberdruckes, des Fülldrucks und der Anzahl der im Prozess erfolgten Befüllvorgänge [3]. Bei gleichbleibendem Produkt, Füllzeit, Umlaufgeschwindigkeit und beaufschlagtem Gasüberdruck ergeben sich annähernd gleiche Filtratmassenströme.

Um ein optimiertes Füllen $\mathrm{zu}$ erreichen, gibt es die Möglichkeit der Resuspendierung. Dabei wird ein bereits gebildetes Partikelhaufwerk zerstört und bei niedriger Froudezahl (Fr) wieder aufgebaut. Die Froudezahl sollte dabei stets kleiner eins sein:

$\mathrm{Fr}=\frac{2 \pi^{2} n^{2} d}{g}<1$

Diese Methode führt jedoch zu dem Problem, dass sich bei schnell filtrierenden Produkten Unwuchten ausbilden können und dass sich die Prozesszeit zwangsläufig verlängert. Der große Vorteil dieser Verfahrensweise ist aber, dass sich Sedimentations- bzw. Entmischungseffekte reduzieren und so ein homogener Produktkuchen gebildet wird. Nach

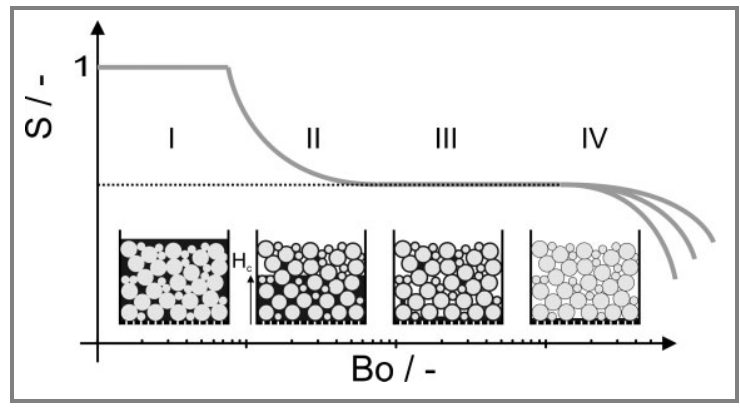

Abbildung 3. Ideales Bonddiagramm bei reiner Zentrifugalkraftterhöhung.

dem eigentlichen Füllvorgang folgt der Prozessschritt der mechanischen Entfeuchtung.

\section{Mechanische Entfeuchtung}

Je effizienter die Flüssigkeit aus dem Kuchen in diesem Prozess entfernt werden kann, desto besser und schneller ist der Produktkuchen abwerfbar und desto weniger Energie ist für den thermischen Trocknungsprozess notwendig $[4,5]$. Wichtige Parameter beim mechanischen Entfeuchten sind die Zentrifugenziffer $C$, der hydraulische Durchmesser $d_{\mathrm{h}}$, die Höhe des Produktkuchens $H_{\mathrm{k}}$, die Oberflächenspannung $\sigma$ und der Benetzungswinkel $\delta$ [6]. Die wichtigsten Kennzahlen, die diese Parameter zusammenfassen, sind folgende Bondzahlen:

$\mathrm{Bo}_{1}=\frac{\rho_{\mathrm{f}} \mathrm{g} C d_{\mathrm{h}} H_{\mathrm{k}}}{\sigma \cos \delta}$

$\mathrm{Bo}_{2}=\frac{\rho_{\mathrm{f}} \mathrm{g} C d_{\mathrm{h}}^{2}}{\sigma \cos \delta}$

Die Bondzahl stellt den Zusammenhang zwischen Zentrifugalkraft und Kapillarkraft dar. Im Bonddiagramm (Abb. 3) ist die Sättigung als Funktion der Bondzahl aufgetragen [7]. Durch die im Experiment erstellten Bonddiagramme kann man für eine definierte Umdrehungszahl eine bestimmte Kuchensättigung voraussagen. Vorraussetzung dafür sind aber konstante Produktwerte für Suspensionsmasse und -konzentration.

Der Filterkuchen entfeuchtet aufgrund der auf die Flüssigkeit wirkenden Zentrifugalkraft. Das Filtermedium hält dabei den Feststoff zurück und bildet so den Filterkuchen. Die Zentrifugalkraft muss die Oberflächenkräfte an den Kapillarhälsen überwinden, um ein Abfließen der Flüssigkeit zu ermöglichen [7]. Das Gleichgewicht der Entfeuchtung ergibt sich aus dem Kräftegleichgewicht zwischen der Gewichtskraft der Flüssigkeitssäule und der Oberflächenkraft, die auf den Umfang der Kapillare wirkt [8]. Die kapillare Steighöhe stellt sich somit in Abhängigkeit der Zentrifugalkraft und des Kapillarradius ein.
Je effizienter die Flüssigkeit aus dem Kuchen entfernt werden kann, desto besser und schneller ist der Produktkuchen abwerfbar und desto weniger Energie ist für den thermischen Trocknungsprozess notwendig. 


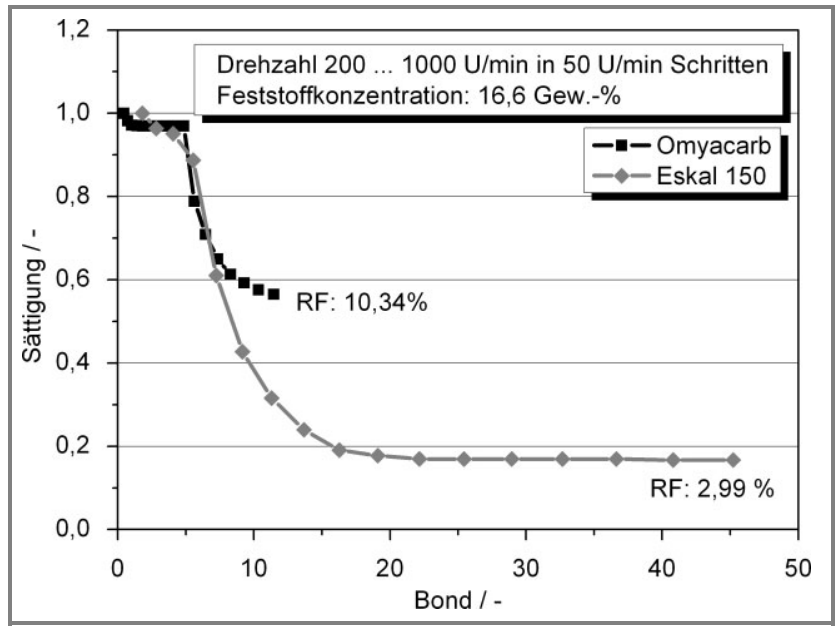

Abbildung 4. Bonddiagramm bei reiner Zentrifugalkrafterhöhung.

Das Bonddiagramm kann in vier charakteristische Bereiche unterteilt werden. Im Bereich I ist der Filterkuchen noch vollständig gesättigt (s. Abb. 3); die kapillare Steighöhe ist gleich der Filterkuchenhöhe [9]. Die Zentrifugalkraft reicht nicht aus, um den kapillaren Eintrittsdruck zu überwinden.

Im Bereich II (s. Abb. 3) sinkt der Flüssigkeitsspiegel in den Filterkuchen hinein; die kapillare Steighöhe ist kleiner als die Filterkuchenhöhe [9]. Oberhalb der kapillaren Steighöhe bleiben Zwickel bestehen. Mit Erhöhung der Zentrifugalkraft sinkt die kapillare Steighöhe, bis die Grobkapillarflüssigkeit vollständig aus dem Filterkuchen entfernt ist. In diesem Bereich kann die Bondzahl als eine reziproke relative Steighöhe der Flüssigkeit aufgefasst werden.

Der Bereich III ist durch das so genannte „Bond-Plateau“ gekennzeichnet. In diesem Bereich liegt keine kapillare Steighöhe mehr vor,

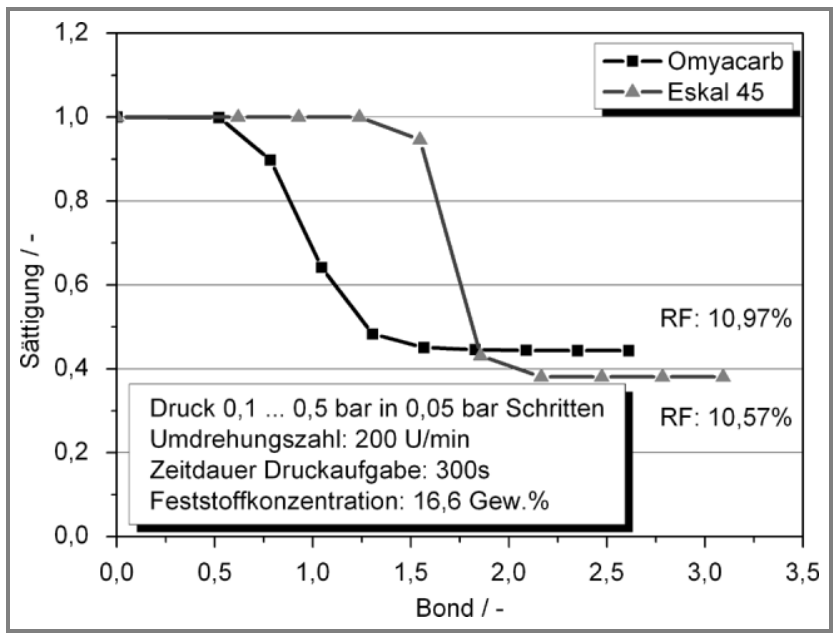

Abbildung 5. Bonddiagramm bei geringer Zentrifugalkraft und Erhöhung des Gasüberdruckes. da die Zentrifugalkraft nicht ausreicht, um die noch mit Flüssigkeit gefüllten Zwickel zu entleeren. Für mindestens eine Größenordnung der $\mathrm{Bo}_{2}$-Zahl bewirkt ein weiterer Anstieg der Zentrifugalkraft keine weitere Zentrifugalentfeuchtung [9]. Bei Material mit rauer Oberflächenstruktur kann das Bond-Plateau leicht geneigt sein, da die Zentrifugalkräfte möglicherweise ausreichen, um die Flüssigkeit aus den Rauigkeiten zu entfernen. Für Produkte mit glatter Oberflächenstruktur liegt das BondPlateau im Bereich von 8 - 10 \% Sättigung. Die Sättigung von oberflächenrauen Produkten kann hingegen auf über $25 \%$ ansteigen.

Für den Bereich IV sind die Zentrifugalkräfte so groß, dass auch die Zwickel entleert werden können [9]. Die $\mathrm{Bo}_{2}$-Zahl beschreibt somit den Bereich IV des Bonddiagramms.

Mit dem Titus-Zentrifugentrockner ist sowohl eine mechanische Entfeuchtung mit reiner Zentrifugalkraft als auch eine Entfeuchtung mit Zentrifugalkraft in Kombination mit überlagertem Gasdruck möglich. Das folgende Diagramm stellt ein Bonddiagramm bei reiner Zentrifugalentfeuchtung dar (s. Abb. 4).

Die Feststoffkonzentration aller Partikelsuspensionen betrug 16,6 Gew.-\% und die Drehzahl wurde dabei in $50 \mathrm{U} / \mathrm{min}$ Schritten von $200 \mathrm{U} / \mathrm{min}$ auf $1000 \mathrm{U} / \mathrm{min}$ gesteigert. Aus sicherheitstechnischen Gründen konnte die Maschine bei der Ermittlung des Bonddiagramms nicht über eine Umdrehungszahl von über $1000 \mathrm{U} / \mathrm{min}$ gefahren werden. Die Sättigung $S$ entspricht dem Wert von eins, wenn der klare Überstand gerade in den Kuchen eintaucht. An diesem Punkt begann die Messung des Filtratgewichtes. Es wurden zu Prozessbeginn keine verfahrenstechnischen Lufteinschlüsse angenommen. Das Gleichgewicht zwischen Kapillar- und Zentrifugalkraft stellte sich ein, sobald der Filtratstrom aus dem Haufwerk bei den jeweiligen Umdrehungszahlen vollständig endete. Demnach ist die jeweilige Filtratmasse bei jeder Gleichgewichtseinstellung $\mathrm{zu}$ ermitteln. Erst dann wurde der nächste Gleichgewichtszustand angefahren. Die gravimetrische Bestimmung des Sättigungsgrades des gesamten Haufwerks erfolgt durch Addition der Filtratmassen. Das Bonddiagramm zeigt, dass sowohl Omyacarb als auch Eskal 150 zu den oberflächenglatten Produkten gehören, da diese bei einer Drehzahl von $1000 \mathrm{U} / \mathrm{min}$ bereits im Bereich des Bondplateaus liegen. Auf Grund der kleineren Kapillaren bei Omyacarb mussten wesentlich größere Zentrifugalkräfte aufgewendet werden, um den gleichen Sättigungsgrad wie bei gröberen Partikelkollektiven zu erzielen.

Für die optimale Reinigbarkeit des Zentrifugentrockners ist ein möglichst trockenes und 
locker aufgebautes Haufwerk vorteilhaft. Feuchtere Produkte führen bei der nachfolgenden Trocknung zu Anhaftungen im Prozessraum. Bei zu hohen Zentrifugalkräften kann der Produktkuchen sogar klumpen [10]. Dadurch erschweren sich sowohl die Trocknung als auch der Austrag und letztendlich auch die Reinigbarkeit der gesamten Maschine.

\section{Drucküberlagerte Zentrifugation}

Neben der klassischen Art der Zentrifugation kann im Titus-Zentrifugentrockner die Flüssigkeit mit Hilfe eines der Zentrifugation überlagerten Gasdruckes aus dem Produkt entfernt werden [11]. In Abb. 5 sind die Ergebnisse der gasdrucküberlagerten Zentrifugation dargestellt.

Bei diesem Versuch blieb die Umdrehungszahl von $200 \mathrm{U} / \mathrm{min}$ konstant. Die Druckerhöhung erfolgte dabei in 0,05-bar-Schritten von 0,1 bar auf 0,5 bar. Jede Druckaufgabe dauerte 300 s. Die Massenkonzentration der Produkte blieb bei 16,6 Gew.-\%. Aus sicherheitstechnischen Aspekten betrug der bei den Versuchen überlagerte Gasdruck maximal 0,5 bar. Wie beim Bonddiagramm mit reiner Zentrifugalkrafterhöhung ist mit dem Eintauchen des Flüssigkeitsüberstands in den Produktkuchen die Sättigung gleich eins. Ab diesem Zeitpunkt begann die Aufzeichnung des Filtratgewichtes. Nach jeder Erhöhung des Gasüberdruckes um 0,05 bar, stellte sich nach einer gewissen Zeit ein Gleichgewicht zwischen Kapillarkraft und Gasüberdruck ein [11]. Über die nach jeder Druckerhöhung anfallende Filtratmasse erfolgte die Ermittlung der Sättigung. Auffallend ist, dass sich mit dem Produkt Omyacarb bei 0,5 bar Gasüberdruck und einer Drehzahl von $200 \mathrm{U} / \mathrm{min}$ ähnliche Entfeuchtungsergebnisse ergeben, wie bei einer Zentrifugation mit $1000 \mathrm{U} / \mathrm{min}$ (s. Abb. 5). Die mechanische Entfeuchtung mit zusätz- lich überlagertem Gasdruck eignet sich besonders für kleinere Partikel, da bei größeren Partikeln schon geringe Zentrifugalkräfte ausreichen, um dem Partikelhaufwerk die Feuchtigkeit zu entziehen. Bei Produkten mit bimodaler Partikelverteilung sind gute Verfahrensergebnisse erreichbar, wenn hohe Zentrifugalkräfte mit hohem Gasüberdruck kombiniert werden [11]. Interessant ist auch, dass sich die jeweiligen Restfeuchten von Omyacarb und Eskal 45 nur sehr gering unterscheiden, obwohl der $x_{50}$-Wert von Eskal 45 bei 43,71 $\mu \mathrm{m}$ und der von Omyacarb bei 76,9 $\mu \mathrm{m}$ liegt (s. Abb. 5). Für beide Stoffe ist erkennbar, dass schon 0,3 bar Gasüberdruck ausreichen, um das „Bondplateau“ zu erreichen. Dieser Prozessschritt vermeidet folglich eine unnötige Produktkompaktierung und erleichtert die thermische Trocknung sowie auch den Produktaustrag. Geringe Anhaftungen und ein optimaler Produktaustrag ermöglichen eine schnelle und effiziente Reinigung.

\section{Kuchenabwurf}

Im Titus-Zentrifugentrockner gibt es die Möglichkeit, den Partikelkuchen mit Hilfe von Gasdruckstößen abzuwerfen (s. Abb. 6) Die Effektivität dieses Prozessschrittes ist direkt vom Füllvorgang und der mechanischen Entfeuchtung abhängig. Dies bedeutet, dass feuchtere oder unregelmäßig aufgebaute Partikelkuchen schwerer abzuwerfen sind als trockene und homogen aufgebaute. Weiterhin ist die Effektivität des Prozesses auch von der Kuchenhöhe, der Partikelgröße und deren Verteilung abhängig (s. Abb. 6).

Vorteil dieser Abwurfweise des Produktkuchens ist, dass keine Grundschicht in der Maschine zurückbleibt. Die Grundschicht neigt bei nachfolgenden Produktzyklen zur Ansammlung von Feinstpartikeln und zur Verdichtung, sodass es zu einem Anstieg der

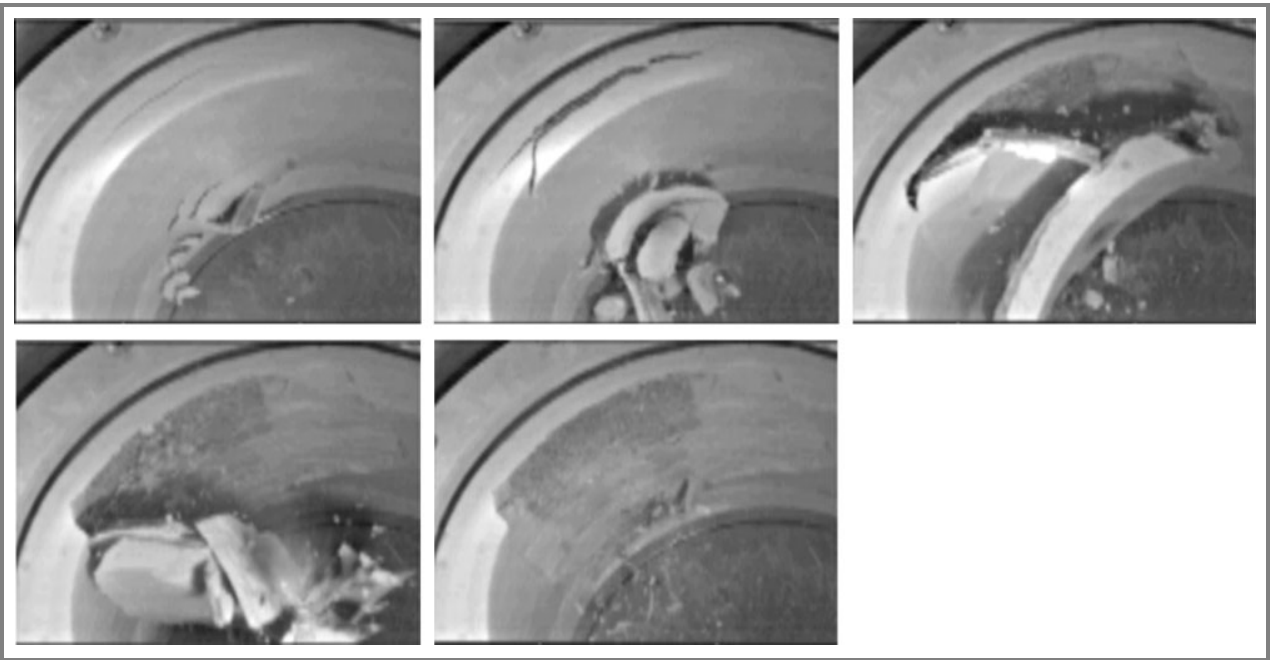

\section{Geringe Anhaftun- gen und ein optima- ler Produktaustrag ermöglichen eine schnelle und effizi- ente Reinigung.}

Abbildung 6. Hochgeschwindigkeitsaufnahmen des Kuchenabwurfs von Eskal 150. 


\section{In dem weiter ent- wickelten Zentrifu- gentrockner wurde die Anzahl der Seg- mente erhöht, um den Abwurf von ge- schichteten Kuchen sicherzustellen.}

kapillaren Steighöhe kommen kann. Bei anderen Maschinen erfolgt der Austrag meist mit Schälwerkzeugen, wobei die Gefahr von Partikelzerkleinerungen besteht, die beimr Abwurf des Kuchens durch Gasdruckstöße nicht gegeben ist. Der Abwurf von monomodalen Produktkuchen, die aus relativ großen Partikeln bestehen, gestaltet sich auch bei höheren Kuchenhöhen als sehr effizient. Bimodale Verteilungen neigen hingegen bei der Zentrifugation zu einer Schichtenbildung. Dies bedeutet, dass aus Gründen der unterschiedlichen Sinkgeschwindigkeit, größere Partikel direkt am Filtergewebe vorzufinden sind und dass kleinere Partikel demnach die Kuchenoberfläche bilden.

Solche Schichten aus feinen Partikeln sind schwerer $\mathrm{zu}$ entfeuchten, wodurch die gemittelte Restfeuchte in diesen Kuchen höher ist als bei monomodalen Partikelgrößenverteilungen. Bei bimodalen Verteilungen bilden sich Klassierschichten aus feinem Material. Dadurch sinkt die Effektivität des Kuchenabwurfs durch Gasdruckstöße. Die Folge davon ist, dass wesentlich mehr Druckstöße notwendig sind, um den Kuchen zu lösen. In dem weiter entwickelten Zentrifugentrockner wurde zusätzlich die Anzahl der Segmente hinter der Trommel erhöht, um den Abwurf von geschichteten Kuchen sicherzustellen.

\section{Absatzweise Trocknung in einer Wirbelschicht mit ideal durchmischten Partikeln \\ Feuchte, rieselfähige Schüttgüter lassen sich in gasdurchströmten Schüttschichten trock- nen. Die Gasgeschwindigkeit sollte für eine homogene Wirbelschicht weit genug über der des Lockerungspunktes liegen. Die Wirbel- schicht ist dann in der Regel gut durchmischt, sodass die Produktfeuchte an jeder Stelle als gleich angenommen werden kann [12].}

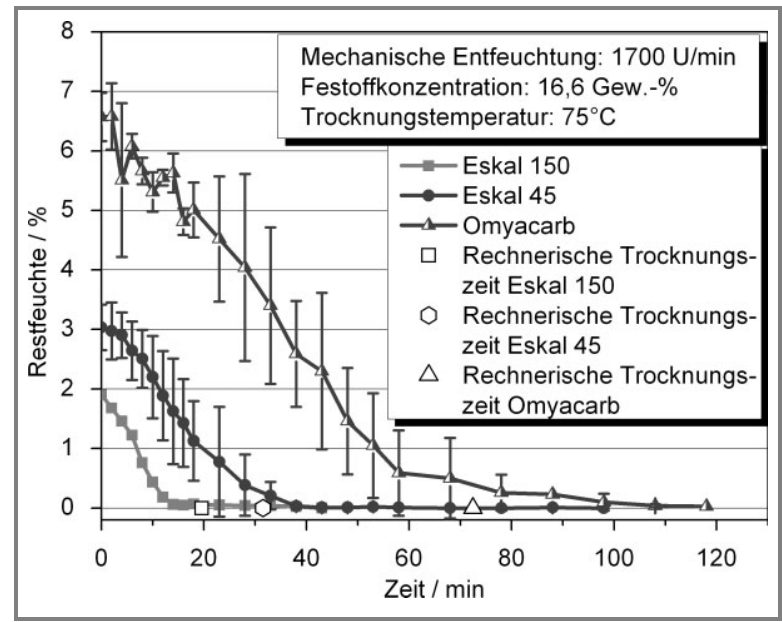

Abbildung 7. Ermittlung der Trocknungsgeschwindigkeit.
Im Folgenden wird die absatzweise Trocknung feuchter Partikel in einer Wirbelschicht betrachtet. Die Produktfeuchte ist in diesem Fall eine Funktion der Zeit und der Strömungsgeschwindigkeit. Die Produkttrockenmasse $M_{\mathrm{S}}$ soll sich dabei in einer Wirbelschicht mit einer Anfangsbeladung $X_{0}$ nach der Zeit $t_{\mathrm{E}}$ auf die Beladung $X_{\mathrm{E}}$ ändern. Die Wirbelschicht wird von dem trockenen Luftmassestrom $\dot{M}_{\mathrm{G}}$ durchströmt, der mit der gleichbleibenden Feuchtebeladung $Y_{\mathrm{e}}$ ein- und mit der vom Fortgang der Trocknung, also der zeitabhängigen Beladung $Y_{\mathrm{a}}(t)$ austritt. Die theoretische Trocknungszeit $t_{\mathrm{E}}$ ergibt sich nach Gnielinski [12] als eine Funktion der dimensionslosen Trocknungszeit $\tau$ und der dimensionslosen Endtrocknungszeit $\tau_{\mathrm{E}}$. Beide Ausdrücke errechnen sich nach folgenden Formeln:

$$
\begin{aligned}
\tau= & \frac{\rho_{\mathrm{g}} \beta\left(Y^{*}-Y_{\mathrm{e}}\right) A}{\left.M_{\mathrm{S}}\left(X_{\mathrm{kr}, \mathrm{I}}-X_{\mathrm{hy}, \mathrm{Gl}}\right)\right)} t \\
\tau_{\mathrm{E}}= & \frac{\zeta_{\mathrm{L}}}{1-\mathrm{e}^{-\zeta_{\mathrm{L}}}\left(\xi_{0}-1\right)+\zeta_{\mathrm{L}}\left(1-\xi_{\mathrm{E}}\right)} \\
& +\ln \frac{1-\mathrm{e}^{-\zeta_{\mathrm{L}}}}{1-\mathrm{e}^{-\zeta_{\mathrm{L}} \xi_{\mathrm{E}}}}
\end{aligned}
$$

Die Trocknungszeit beträgt demnach:

$t_{\mathrm{E}}=\frac{\tau_{\mathrm{E}}}{\tau}$

Die Berechnung der theoretischen Trocknungszeit für Omyacarb von $67 \mathrm{~min}$, bei Eskal 45 von 34 min und bei Eskal 150 von 20 min folgt aus Gl. (7). Diese errechneten Trocknungszeiten zeigen eine gute Übereinstimmung mit experimentell bestimmten Werten (s. Abb. 7). Bei maximaler Umdrehungszahl von $1700 \mathrm{U} / \mathrm{min}$ sind Restfeuchten bei Omyacarb von $6,5 \%$ erreichbar. Eskal 45 weist für diese Zentrifugalkraft eine Restfeuchte von $3 \%$ und Eskal 150 eine Restfeuchte von 1,9\% auf. Aus Abb. 7 ist erkennbar, dass beim Trocknungsprozess durch Gaspulsation, Restfeuchten von unter $1 \%$ auch bei einem bimodalen Produkt wie Omyacarb in einem Zeitraum von unter 60 min realisierbar sind. Die Trocknung von monomodalen Produkten benötigt in der Regel weniger Zeit, im Vergleich zu Produkten mit bimodaler Partikelgrößenverteilung. Der höhere Anteil des Feinguts und auch der nach der mechanischen Entfeuchtung höhere Feuchteanteil erklärt die längere Trocknungsdauer von Omyacarb.

Omyacarb weist in der Anfangsphase des Trockungsprozesses in der Restfeuchte eine relativ starke Schwankung auf. Dies kann durch die bimodale Partikelgrößenverteilung erklärt 
werden, da feinere Partikelkollektive nach der mechanischen Entfeuchtung eine höhere Feuchte haben als gröbere Partikel. Das bedeutet, dass auch Proben entnommen werden können, die einen höheren Feuchtegehalt aufweisen, da das Haufwerk nicht ideal durchmischt ist.

Aus den charakteristischen Trocknungskennlinien erfolgt mit Hilfe von Ausgleichsgeraden die Ermittlung der Trocknungsgeschwindigkeit der jeweiligen Produkte, die sich aus der Steigung der Geraden ergibt. Die Trocknungsgeschwindigkeiten aller Produkte waren im Rahmen der Messgenauigkeit nahezu identisch.

Wirbelschicht-Zustandsdiagramm nach Reh Bei einer Wirbelschicht werden charakteristische Grundeigenschaften und -vorgänge ausgenutzt [13]. Diese sind:

- Vermischung von Partikeln,

- hohe Relativgeschwindigkeiten zwischen fluider und dispers fester Phase,

- häufige Partikel/Partikel und Partikel/ Wand-Stöße.

Für durchströmte Schüttschichten hat Reh [13] ein Diagramm entwickelt, in dem homogene und inhomogene Strömungszustände von Wirbelschichten dargestellt werden können. Wie aus Abb. 8 zu erkennen ist, bildet die Äquiporositätslinie für $\varepsilon=0,4$ die Grenze zwischen Festbett und Wirbelschicht [13]. Dieser Wert wird auch pauschal als Lockerungsporosität angenommen. Der Wirbelschichtbereich erstreckt sich von $\varepsilon \geq 0,4$ (Festbett) bis $\varepsilon \rightarrow 1$. Oberhalb von $\varepsilon=1$ erfolgt der Partikelaustrag. Mit dem Zustandsdiagramm lassen sich näherungsweise die Gasgeschwindigkeiten abschätzen. Die Kennlinien [13] des Diagramms verwenden folgende dimensionslose Kennzahlen:

\section{Archimedeszahl:}

$\operatorname{Ar}=\frac{\Delta \rho \mathrm{gd}_{\mathrm{P}}^{3}}{v^{2} \rho_{\mathrm{G}}}=\frac{1}{K}$

$\Omega$-Zahl: $\quad \Omega=\frac{\rho_{\mathrm{g}} u^{3}}{\Delta \rho v \mathrm{~g}}=M$

Reynoldszahl: $\quad \operatorname{Re}_{\mathrm{P}}=\frac{u d_{\mathrm{P}}}{v}$

Froudezahl: $\quad F r_{\mathrm{P}}^{2}=\frac{u^{2}}{\mathrm{~g} d_{\mathrm{P}}}$

Dabei sind die Froudezahl und die Reynoldszahl nicht voneinander unabhängig, da beide die Parameter Trägergeschwindigkeit $u$ und Partikeldurchmesser $d_{\mathrm{P}}$ beinhalten.

Es gilt bei der Elimination der Trägergasgeschwindigkeit:

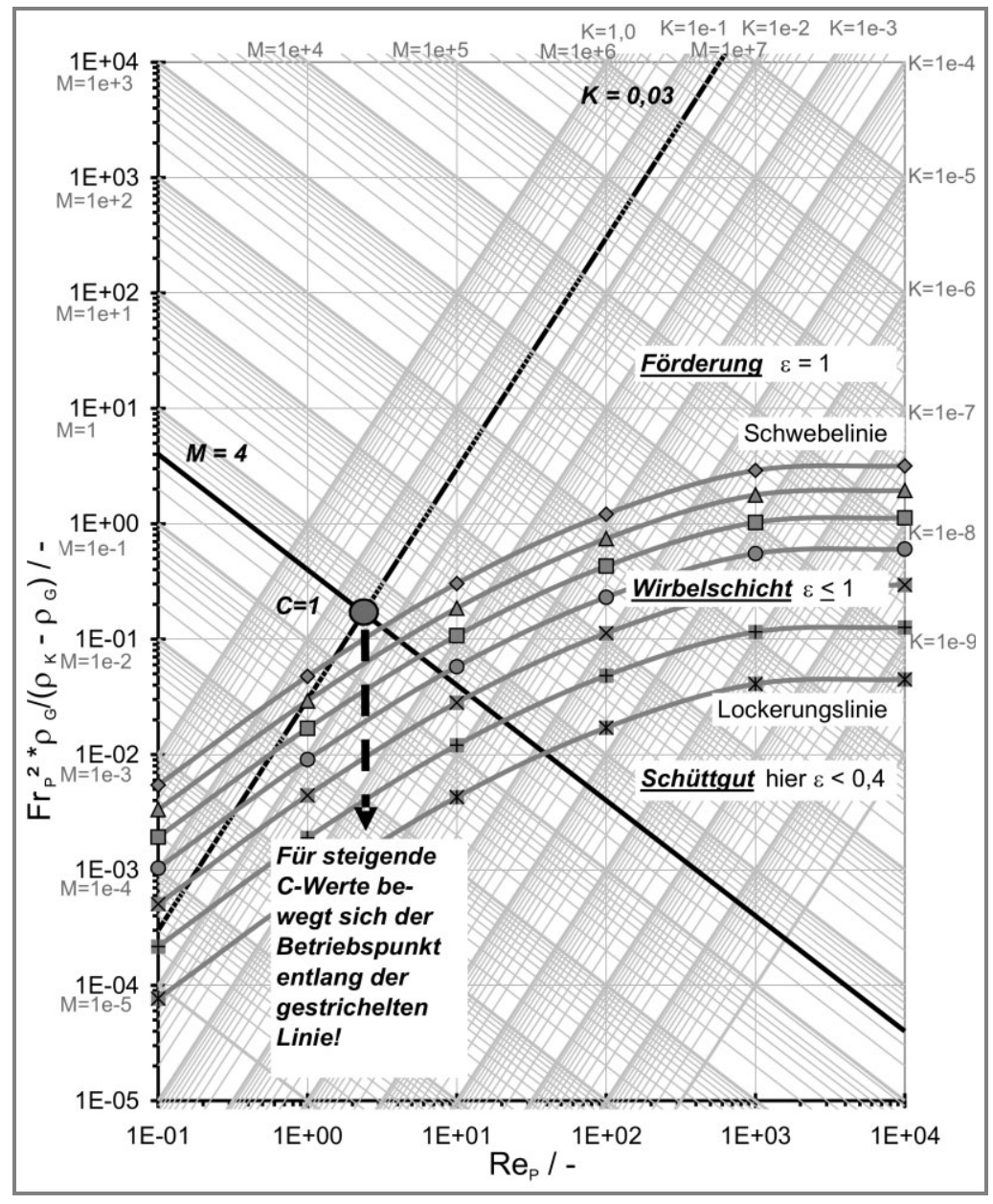

Abbildung 8. Betriebspunkt des Zentrifugentrockners im Zustandsdiagramm für eine Gas/Feststoff-Wirbelschicht.

$$
\begin{aligned}
& \operatorname{Fr}_{\mathrm{P}}^{2} \frac{\rho_{\mathrm{G}}}{\rho_{\mathrm{P}}-\rho_{\mathrm{G}}}=\frac{u^{2}}{d_{\mathrm{P}} g} \frac{\rho_{\mathrm{G}}}{\rho_{\mathrm{P}}-\rho_{\mathrm{G}}}= \\
& \frac{v^{2}}{d_{\mathrm{P}}^{3} \mathrm{~g}} \frac{\rho_{\mathrm{G}}}{\rho_{\mathrm{P}}-\rho_{\mathrm{G}}} \operatorname{Re}^{2}
\end{aligned}
$$

Zustandsänderungen können längs einer parallelen Geradenschar durch den Parameter $K$ (s. Gl. (8)) definiert werden $[13,14]$. Zur $K$ Wert-Ermittlung spielen die Stoffeigenschaften des Feststoffs, wie Teilchendurchmesser, Dichte und die Stoffeigenschaften des strömenden Mediums, wie die kinematische Zähigkeit und die Dichte eine wesentliche Rolle. In der Staub- und Wirbelschichttechnik ergeben sich Anwendungsbereiche zwischen $0,1<\operatorname{Re}<10^{4}$. Daraus folgt ein $K$-Wertebereich von $1,0>K>10^{-10}$ [15]. Betrachtet man den Einfluss von Korngemischen auf die Wirbelschicht, so ist es sinnvoll, durch Umformung und Erweiterung die Reynoldszahl mit der Froudezahl zu verknüpfen. Im Zustandsdiagramm ist dies ebenfalls eine parallele Geradenschar, analog den Linien $K=$ konst. Bei zunehmender Geschwindigkeit der Zweistoffströmung disperser Partikel, ändert sich die Widerstandsfunktion im Diagramm längs der 
Der neuste Typ des Titus-Zentrifugentrockners $\mathbf{4 0 0}$ ist mit einem neuen Trocknungsverfahren ausgestattet, das die Trocknungsgeschwindigkeit erhöht und gleichzeitig den Austrag des Feinanteils vermindert.
Linie $K=$ konst. [13]. Dieser Zusammenhang bleibt durch die drei Bereiche Schüttgut, Wirbelschicht und Förderung erhalten. Durch Variation des Partikeldurchmessers bei gegebener Trägergasgeschwindigkeit $u$ ändert sich die Widerstandsfunktion längs einer Geraden $M=$ konst. (s. Gl. (9)). Im Zustandsdiagramm bilden die dimensionslosen Parameter $K$ und $M$ ein Koordinatensystem, wodurch jeder Strömungszustand darstellbar ist [13]. Durch die angelegte Zentrifugalkraft verschiebt sich die Lage der $\Omega$ - und Archimedeszahl, da diese Werte von der Gravitation abhängen. Das sekundäre Koordinatensystem muss dann für jede Zentrifugenziffer erneut konstruiert werden. Dieses Verfahren ermöglicht folglich die Wirbelschicht sowohl durch die Gasgeschwindigkeit der Schussdüsen als auch über die Zentrifugalbeschleunigung bei feinkörnigen Partikeln zu kontrollieren und das Produkt so sicher zu trocknen. Durch die angelegte Zentrifugalkraft vermindert sich der Austrag des Feinanteils in diesem Verfahren, da die Porosität der Wirbelschicht unterhalb der Austragslinie bleibt. Die Reinigung des Filtergewebes der Trommel vereinfacht sich, da die Partikel nicht in das Filtergewebe der Trommel gelangen.

\section{Reinigung}

Da bei verfahrenstechnischen Maschinen und Apparaten, die in der Pharmaindustrie eingesetzt werden, ein wesentlicher Teil der Produktionszeit auf die Reinigung entfällt, ist die Reinigung von höchster Wichtigkeit für die Gesamtprozesszeit [15]. Der Titus-Zentrifugentrockner 400 SD im Pharmadesign (Sterildesign) verfügt über drei produktberührte Bereiche, die unabhängig von einander reinigbar und entsprechend validierbar sind. Dazu gehören der eigentliche Verfahrensraum mit der Filtertrommel, der Ringkanal zum Feststoffaustrag und das Stauscheibengehäuse. Diese Bereiche verfügen jeweils über ein eigenes (Cleaning-In-Place) CIP-Düsensystem mit Ablauf (s. Abb. 9). Darüber hinaus verfügt die Zentrifuge über konstruktive Details, wie gas-

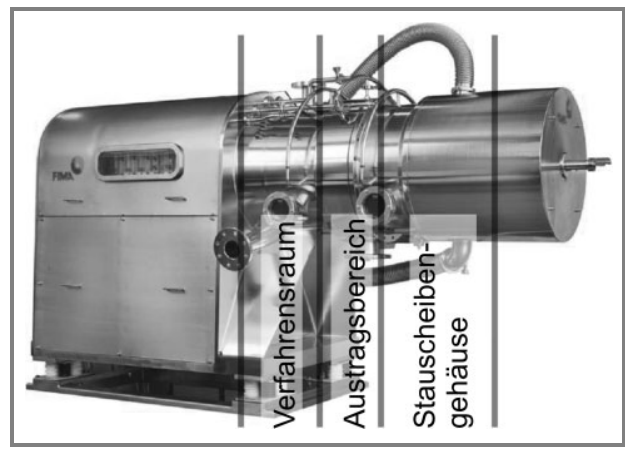

geschmierte Gleitringdichtungen, Schaugläser an schwer zugänglichen Stellen und die Vermeidung unnötiger Einbauten in den Verfahrensraum. Bevor die In-Place-Reinigung beginnt, ist es von großer Wichtigkeit, dass die vorangegangenen Prozessschritte in der bereits beschriebenen Weise aufeinander abgestimmt und optimiert sind.

Zur Bestätigung der Reinigungsfähigkeit des Filtergewebes wurde ein spezieller Reinigungstest entwickelt [16], der die Bewertung der Reinigungsfähigkeit von mehrlagigen Metallfiltergeweben ermöglicht. Dieser dreistufige, indirekte Reinigungstest besteht aus der Kontamination des Filtermediums mit Sporen, einer definierten Reinigung und einem Restverunreinigungsnachweis nach der Inkubation [17]. Die Reinigungstests führten zu einer Auswahl an Filtermedien, die durch ihre spezielle Anordnung an Stützgeweben das beste Reinigungsergebnis lieferten.

\section{Das „Gegenimpulsverfahren“ des TZT 400}

Der neuste Typ des Titus-Zentrifugentrockners 400 ist mit einem neuen Trocknungsverfahren ausgestattet. Bei diesem Prozessschritt gelang es, die Trocknungsgeschwindigkeit zu erhöhen und gleichzeitig den Austrag des Feinanteils $\mathrm{zu}$ vermindern. Im Folgenden soll schematisch der Unterschied zwischen dem klassischen Verfahren und dem Gegenimpulsverfahren dargestellt werden.

In Prozessen mit bimodalen Partikelgrößenverteilungen bildet sich auf der Oberseite des Produktkuchens eine aus Feingut bestehende Deckschicht aus. Bei der mechanischen Entfeuchtung muss ein über der Deckschicht stehender Überstand durch diese feine Kuchenoberfläche drainieren. Die Partikeldeckschicht bestimmt so im klassischen Verfahrensprozess die Filtratstrommenge [6]. Im neuen Prozess gibt es schon während der Trocknung Gegenimpulse mit Gas bzw. Luft. Dadurch steigt die Permeabilität des Produktkuchens. Die Folge ist eine verbesserte Filtratleistung. Bei der klassischen Wirbelschichttrocknung durchströmt das Heißgas das Produkt nur einmal, wodurch Ablagerungen in der Trommel nur unzureichend getrocknet werden können. Das neue Gegenimpulsverfahren bietet den Vorteil, dass sich die Auftriebskraft durch die Druckstöße und die Zentrifugalkraft auf ein Partikel unabhängig voneinander einstellen lassen. Das Trocknungsgas durchströmt in diesem Verfahrensschritt das Haufwerk zweimal. Durch dieses Verfahren minimiert sich der Austrag des Feinanteils, da der Feinanteil im Haufwerk 
durch Tiefenfiltration im Prozessraum bleibt. Einen genaueren Eindruck des Verfahrens vermitteln die Hochgeschwindigkeitsaufnahmen (s. Abb. 10). Hier wird ein kurzer Druckimpuls bei einer Drehzahl von 120 U/min in die unteren beiden Kammern injiziert. Die Trommel dreht sich dabei um $90^{\circ}$.

Dieses Trocknungsverfahren verkürzt auch die Trocknungszeit, da zusätzliches Trocknungsgas über die Füllwelle in die Filtertrommel eingeleitet werden kann. Die gleichmäßigere Durchströmung des Produktkuchens mit Trocknungsgas ist in diesem Prozessschritt aufgrund der höheren Porosität gewährleistet. Die Auflockerung ermöglicht eine effektivere Ausnutzung des Trocknungsgases. Gleichzeitig wird eine Agglomeratbildung des Produktes verhindert. Durch die Verhinderung des Feinpartikelaustrags gelangen außerdem weniger Partikel in das Filtergewebe. Die Reinigung ist dadurch wesentlich schneller und effizienter, da es keine Filtergewebeverstopfungen durch Produktpartikel geben kann.

\section{Zusammenfassung}

Alle Prozessschritte nehmen direkten Einfluss auf die Reinigung des Zentrifugentrockners. Die Bedeutung des Befüllvorganges konnte anhand von extremen Befülldrücken und des daraus resultierenden Haufwerksaufbaus verdeutlicht werden. Bei Produkten die zur Sedimentation bzw. zur Entmischung neigen, ergaben sich die besten Resultate durch Resuspendierung des bereits gebildeten $\mathrm{Ku}-$ chens. Die Beurteilung der Zentrifugal- bzw. der drucküberlagerten Entfeuchtung erfolgt durch ein Bonddiagramm. Betrachtet man die Sättigung über der Bondzahl, dem Verhältnis von Massenkräften zu den Kapillarkräften, so gelingen selbst an der Pilotanlage Aussagen über die optimale Drehzahl und den benötigten Gasüberdruck. Die Auswahl der optimalen Entfeuchtungsparameter ist nun auch bei breit verteilten Produkten möglich. Der Abwurf des Produkthaufwerks erfolgt im klassischen Verfahren durch Druckstöße in die segmentierte Filtertrommel. Ein vollständiger Abwurf ist für einen geringen Produktverlust und ein gutes Reinigungsergebnis ebenfalls essentiell. Hochgeschwindigkeitsaufnahmen veranschaulichten die Vorteile dieses restschichtfreien Abwurfs besonders in der überarbeiteten Maschine, da sie mit einer höheren Anzahl an Kammern ausgestattet ist. In Abhängigkeit der jeweiligen Betriebsparameter erfolgte die Ermittlung der genauen Lage der Wirbelschicht im Zustandsdiagramm nach Reh. Der Trocknungsverlauf ermöglichte sowohl eine experi-

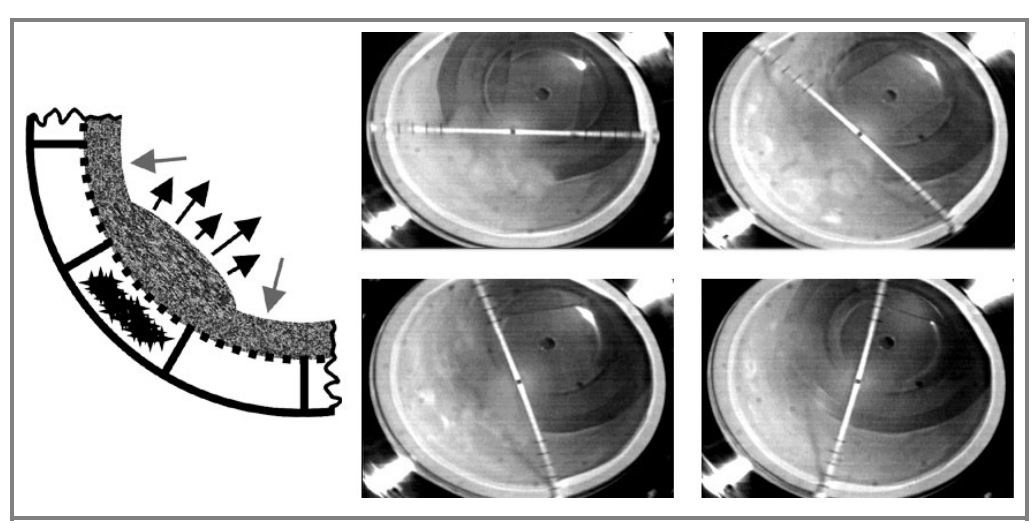

Abbildung 10. Links: Schematischer Darstellung des Druckstoßes in eine Kammer; Rechts: Hochgeschwindigkeitsaufnahmen des Gegenimpulsverfahrens. Nach einer viertel Umdrehung liegt das Haufwerk wieder an der Trommelwand an.

mentelle als auch eine theoretische Bestimmung der Trocknungsgeschwindigkeit und der Gesamttrocknungszeit. Die Dokumentation des neuen Gegenimpulsverfahrens gelang anhand von Hochgeschwindigkeitsaufnahmen. Durch die Verhinderung des Feinpartikelaustrags beim Gegenimpulsverfahren erfolgte die Reinigung wesentlich schneller und effizienter. Die genaue Abstimmung der einzelnen Verfahrensschritte untereinander ist bei komplexen „multi-purposen“ Anlagen von entscheidender Bedeutung, um auch bei hochaktiven Produkten höchste Reinigungsanforderungen zu erfüllen.

\section{Dipl.-Ing. Sebastian Stahl}

(Sebastian.Stahl@mvm.uni-karlsruhe.de),

Johannes Ruland,

Prof. Dr.-Ing habil. Hermann Nirschl,

Mechanische Verfahrenstechnik und Mechanik,

Universität Karlsruhe (TH), Am Forum 8,

D-76131 Karlsruhe, Germany.

\section{Formelzeichen}

$\begin{array}{lll}A & {\left[\mathrm{~m}^{2}\right]} & \text { Oberfläche } \\ \mathrm{Bo}_{1} & {[-]} & \text { Bondzahl 1 } \\ \mathrm{Bo}_{2} & {[-]} & \text { Bondzahl 2 } \\ C & {[-]} & \text { C-Wert } \\ d_{\mathrm{P}} & {[\mathrm{m}]} & \text { Partikeldurchmesser } \\ d_{\mathrm{h}} & {[\mathrm{m}]} & \text { hydraulischer Durchmesser } \\ g & {\left[\mathrm{~m} / \mathrm{s}^{2}\right]} & \text { Erdbeschleunigung } \\ H_{\mathrm{K}} & {[\mathrm{m}]} & \text { Kuchenhöhe } \\ M_{\mathrm{S}} & {[\mathrm{kg}]} & \text { Masse } \\ \dot{M g} & {[\mathrm{~kg} / \mathrm{s}]} & \text { Massenstrom Gas } \\ n & {[\mathrm{~mol}]} & \text { Mol } \\ n_{\mathrm{dr}} & {[1 / \mathrm{s}]} & \text { Umdrehungszahl } \\ p & {[\mathrm{bar}]} & \text { Partialdruck } \\ R_{\mathrm{S}} & {[-]} & \text { Restfeuchte } \\ S & {[-]} & \text { Sättigung } \\ t_{\mathrm{E}} & {[\mathrm{s}]} & \text { Zeit (Ende) } \\ u & {[\mathrm{~m} / \mathrm{s}]} & \text { Geschwindigkeit }\end{array}$




\begin{tabular}{|c|c|c|}
\hline$X_{\text {hy,Gl }}$ & {$[-]$} & $\begin{array}{l}\text { Gleichgewichtsfeststoffbela- } \\
\text { dung Hydrat }\end{array}$ \\
\hline$X_{\text {krit }}$ & {$[-]$} & kritische Feststoffbeladung \\
\hline$X_{0}$ & {$[-]$} & Anfangsbeladung \\
\hline$Y_{0}$ & {$[-]$} & Ausgangsbeladung \\
\hline$Y^{*}$ & {$[-]$} & $\begin{array}{l}\text { Sättigungsbeladung der } \\
\text { Luft }\end{array}$ \\
\hline$Y_{\mathrm{e}}$ & {$[-]$} & Luftbeladung Eingang \\
\hline$x_{50,3}$ & {$[\mathrm{~m}]$} & $\begin{array}{l}\text { Medianwert der Massen- } \\
\text { summenverteilung }\end{array}$ \\
\hline$x_{\mathrm{P}}$ & {$[\mathrm{m}]$} & Partikeldurchmesser \\
\hline$z$ & {$[\mathrm{~m}]$} & $\begin{array}{l}\text { Laufkoordinate (Ursprung } \\
\text { am Filtermedium) }\end{array}$ \\
\hline$\beta$ & {$[\mathrm{m} / \mathrm{s}]$} & $\begin{array}{l}\text { Stoffübertragungs- } \\
\text { koeffizient }\end{array}$ \\
\hline$\delta$ & {$\left[{ }^{\circ}\right]$} & Kontaktwinkel \\
\hline$\varepsilon$ & {$[-]$} & Kuchenporosität \\
\hline$\zeta_{0}$ & {$[-]$} & $\begin{array}{l}\text { Dimensionslose Wirbel- } \\
\text { schichthöhe } z=0\end{array}$ \\
\hline$\zeta_{\mathrm{E}}$ & {$[-]$} & $\begin{array}{l}\text { Dimensionslose Wirbel- } \\
\text { schichthöhe am Ende der } \\
\text { Trocknung }\end{array}$ \\
\hline$\zeta_{\mathrm{L}}$ & {$[-]$} & $\begin{array}{l}\text { Dimensionslose Wirbel- } \\
\text { schichthöhe } z=L\end{array}$ \\
\hline$\vartheta$ & {$\left[{ }^{\circ} \mathrm{C}\right]$} & Temperatur \\
\hline$\rho_{\mathrm{g}}$ & {$\left[\mathrm{kg} / \mathrm{m}^{3}\right]$} & Dichte Trägergas \\
\hline$\rho_{\mathrm{P}}$ & {$\left[\mathrm{kg} / \mathrm{m}^{3}\right]$} & Dichte Partikel \\
\hline$\rho_{\mathrm{f}}$ & {$\left[\mathrm{kg} / \mathrm{m}^{3}\right]$} & Dichte Fluid \\
\hline$\tau$ & {$[\mathrm{s}]$} & $\begin{array}{l}\text { Dimensionslose Tock- } \\
\text { nungszeit }\end{array}$ \\
\hline$\tau_{\mathrm{E}}$ & {$[\mathrm{s}]$} & Endtocknungszeit \\
\hline$v$ & {$\left[\mathrm{~m}^{2} \mathrm{~s}^{-1}\right]$} & Kinematische Viskosität \\
\hline$\sigma$ & {$[\mathrm{N} / \mathrm{m}]$} & Oberflächenspannung \\
\hline$\xi_{0}$ & {$[-]$} & $\begin{array}{l}\text { dimensionslose Guts- } \\
\text { feuchte zum Zeitpunkt } 0\end{array}$ \\
\hline$\xi_{\mathrm{E}}$ & {$[-]$} & $\begin{array}{l}\text { dimensionslose Guts- } \\
\text { feuchte am Ende (Zeit) }\end{array}$ \\
\hline
\end{tabular}

\section{Literatur}

[1] F. Reif, M. Wünsch, H. König, W. Stahl, Aufbereit. Tech. 1990, 31 (3), 117.

[2] A. Ettmayr, G.Bickert, W. Stahl, F\&S Filtr. Sep. 2001, 15 (2), 58.

[3] G. Mayer, Dissertation, Universität Karlsruhe (TH) 1986.

[4] B. Nill, Chem. Ing. Tech. 1986, 58 (9), 720.

[5] A. Rushton, M. Spear, Filtration 1975, 12 (3), 254.

[6] P. Iohn, Aufbereit. Tech. 1967, 8 (11), 615.

[7] H. Schubert, Dissertation, Universität Karlsruhe (TH) 1972.

[8] C. Alt, Filtration, in Ullmanns Encyclopädie der technischen Chemie, 4.Auflage, Band 2, VCH, Weinheim 1984, 154.

[9] W. Stahl, Industrie - Zentrifugen. Fest- FlüssigTrennung, Band II, 1.Auflage, DrM Press, Männedorf 2004.

[10] H. W. Müller, J. Schaffer, S. Conrad, Chem. Tech. 1980, 32 (2), 76.

[11] C. Stadager, Dissertation, Universität Karlsruhe (TH) 1995.

[12] V. Gnielinski, A. Mersmann, F. Thurner, Verdampfung, Kristallisation, Trocknung, Vieweg Verlag, Braunschweig 1993.

[13] L. Reh, Dissertation, Universität Karlsruhe (TH) 1962.

[14] F. Schytil, Mitt. Arbeitsbereich - Metallgesellschaft AG 1959, 1.

[15] S. Rippberger, FQS Filtr. Sep. 2004, 18, 262.

[16] S. Stahl, S. Siggelkow, H. Nirschl, Eng. Life Sci. 2007, 7 (2), 136. DOI: 10.1002/elsc.200620183

[17] S. Ripperger, FQS Filtr. Sep. 2004, 3, 110. 